\title{
SHELLABILITY AND HIGHER COHEN-MACAULAY CONNECTIVITY OF GENERALIZED CLUSTER COMPLEXES
}

\author{
CHRISTOS A. ATHANASIADIS AND ELENI TZANAKI
}

\begin{abstract}
Let $\Phi$ be a finite root system of rank $n$ and let $m$ be a nonnegative integer. The generalized cluster complex $\Delta^{m}(\Phi)$ was introduced by S. Fomin and N. Reading. It was conjectured by these authors that $\Delta^{m}(\Phi)$ is shellable and by V. Reiner that it is $(m+1)$-Cohen-Macaulay, in the sense of Baclawski. These statements are proved in this paper. Analogous statements are shown to hold for the positive part $\Delta_{+}^{m}(\Phi)$ of $\Delta^{m}(\Phi)$. An explicit homotopy equivalence is given between $\Delta_{+}^{m}(\Phi)$ and the poset of generalized noncrossing partitions, associated to the pair $(\Phi, m)$ by D. Armstrong.
\end{abstract}

\section{INTRODUCTION}

Let $\Phi$ be a finite root system of rank $n$ and let $m$ be a nonnegative integer. The generalized cluster complex $\Delta^{m}(\Phi)$ was introduced by S. Fomin and N. Reading [1], soon after it was studied in [19] in the special cases in which $\Phi$ has type $A$ or $B$ in the Cartan-Killing classification. If $\Phi$ has type $A$, then faces of $\Delta^{m}(\Phi)$ can be described in terms of polygonal subdivisions of a convex polygon with $m(n+1)+2$ vertices (see Section 2.2). If $m=1$ and $\Phi$ has type $A$, then $\Delta^{m}(\Phi)$ is combinatorially isomorphic to the boundary complex of the $n$-dimensional simplicial associahedron, a classical object of study in enumerative and polyhedral combinatorics. More generally, $\Delta^{m}(\Phi)$ reduces to the cluster complex $\Delta(\Phi)$ when $m=1$, a simplicial complex of importance in the context of cluster algebras and $Y$-systems [12, 13]; see [10] for a nice introduction to these topics.

Motivation for defining and studying generalized cluster complexes came from at least two directions. In [11 combinatorial algorithms for determining Coxeter theoretic invariants were given, in which certain identities satisfied by the face numbers of generalized cluster complexes are crucial. On the other hand, it was conjectured by the first author that the $m$-generalized Narayana (or Fuss-Narayana) numbers defined in [3] for a crystallographic root system $\Phi$ form the $h$-vector of an $(n-1)$-dimensional Cohen-Macaulay simplicial complex. It follows from the results of this paper (as well as those of [3, 11]) that the generalized cluster complex $\Delta^{m}(\Phi)$ is the desired complex having these properties (this was verified earlier by the second author in the special cases of [19]). Moreover, it has been shown [2, 6, 11, 19, 20] that the complex $\Delta^{m}(\Phi)$, as well as a natural subcomplex $\Delta_{+}^{m}(\Phi)$ called its positive part, share essentially all enumerative properties of cluster complexes, relating to a variety of interesting structures within the algebraic combinatorics of Coxeter groups, root systems and hyperplane arrangements; see [3] [10, Lecture 5] [11].

Date: July 3, 2006; revised, February 27, 2007.

2000 Mathematics Subject Classification. Primary 20F55; Secondary 05E99, 13F55, 52B22, $55 \mathrm{U} 10$. 
This paper shows that the complexes $\Delta^{m}(\Phi)$ and $\Delta_{+}^{m}(\Phi)$ have attractive topological properties as well. Recall that a simplicial complex $\Delta$ is said to be $k$-CohenMacaulay (over $\mathbb{Z}$ or some field $\mathbb{K}$ ) if the complex obtained from $\Delta$ by removing any subset of its vertex set of cardinality less than $k$ is Cohen-Macaulay (over $\mathbb{Z}$ or $\mathbb{K})$ and has the same dimension as $\Delta$. The following theorem is the main result of this paper.

Theorem 1.1. $\quad$ (i) The simplicial complex obtained from $\Delta^{m}(\Phi)$ by removing any subset of its vertex set of cardinality not exceeding $m$ is pure, of dimension $n-1$, and shellable. In particular, $\Delta^{m}(\Phi)$ is shellable, hence homotopy equivalent to a wedge of $(n-1)$-dimensional spheres, and $(m+1)$-CohenMacaulay.

(ii) The simplicial complex obtained from $\Delta_{+}^{m}(\Phi)$ by removing any subset of its vertex set of cardinality not exceeding $m-1$ is pure, of dimension $n-1$, and shellable. In particular, $\Delta_{+}^{m}(\Phi)$ is pure of dimension $n-1$ and shellable, hence homotopy equivalent to a wedge of $(n-1)$-dimensional spheres, and m-Cohen-Macaulay.

A few comments on the theorem are in order. Shellability of $\Delta^{m}(\Phi)$ was conjectured by S. Fomin and N. Reading [11, Conjecture 11.3] (see also [1, Problem 6.3]) and verified by the second author [19] when $\Phi$ has type $A$ or $B$. This conjecture was extended to $\Delta_{+}^{m}(\Phi)$ by the authors [6, Conjecture 4.6]. The question of higher Cohen-Macaulay connectivity of $\Delta^{m}(\Phi)$ was raised by V. Reiner; see 1, p. 17] (later, the same question was raised independently by G. Kalai [14]). The result of Theorem 1.1 in this respect is best possible (see Remark 3.2) and nontrivial even when $\Phi$ has type $A$. The Euler characteristic of $\Delta^{m}(\Phi)$ was computed in [11, Section 11]. It is known [11, Section 10] that the $m$-generalized Narayana numbers defined in [3] for crystallographic $\Phi$ coincide with the entries of the $h$-vector of $\Delta^{m}(\Phi)$, except possibly when $m \geq 2$ and $\Phi$ contains an irreducible component of type $F_{4}, E_{6}, E_{7}$ or $E_{8}$. Hence the statement on shellability in Theorem 1.1 (i) establishes the conjecture on these numbers, mentioned earlier, in these cases.

The concept of higher Cohen-Macaulay connectivity was introduced by K. Baclawski [7. We refer the reader to [7] [16] [17, Section III.3] and references given in these sources for other interesting classes of simplicial complexes known to be $k$-Cohen-Macaulay for some $k \geq 2$.

The general layout of this paper is as follows. Section 2 includes background on simplicial complexes, (generalized) cluster complexes and a related partial order on a finite real reflection group. In particular, a new characterization (Theorem 2.3) of $\Delta^{m}(\Phi)$, due to the second author [20, 21], is reviewed, generalizing the one for $\Delta(\Phi)$ given by T. Brady and C. Watt [9, Section 8]. The proof of Theorem 1.1, which relies on this characterization, is given in Section 3. That section includes also a computation of the Euler characteristic of $\Delta_{+}^{m}(\Phi)$ (Corollary 3.3). Finally some applications to the topology of the posets of generalized noncrossing partitions, introduced and studied by D. Armstrong [2, are given in Section 4 .

\section{BACKGROUND}

Throughout the paper we denote by $|\sigma|$ the cardinality of a finite set $\sigma$.

2.1. Simplicial complexes. Let $E$ be a finite set. An (abstract) simplicial complex on the ground set $E$ is a collection $\Delta$ of subsets of $E$ such that $\tau \subseteq \sigma \in \Delta$ 
implies $\tau \in \Delta$. The set $V=\{v \in E:\{v\} \in \Delta\}$ is the set of vertices of $\Delta$. The elements of $\Delta$ are called faces and those which are maximal with respect to inclusion are called facets. The dimension of a face $\sigma$ is defined as one less than the cardinality of $\sigma$ and the dimension of $\Delta$ as the maximum dimension of a face. The complex $\Delta$ is pure if all its facets have the same dimension and flag if all its minimal nonfaces have two elements. The $k$-skeleton $\Delta^{\leq k}$ of $\Delta$ is the subcomplex formed by the faces of $\Delta$ of dimension at most $k$. The simplicial join $\Delta_{1} * \Delta_{2}$ of two abstract simplicial complexes $\Delta_{1}$ and $\Delta_{2}$ on disjoint ground sets has as its faces the sets of the form $\sigma_{1} \cup \sigma_{2}$, where $\sigma_{1} \in \Delta_{1}$ and $\sigma_{2} \in \Delta_{2}$.

The link of $v \in E$ in $\Delta$ is defined as $\operatorname{lk}_{\Delta}(v)=\{\sigma \backslash\{v\}: \sigma \in \Delta, v \in \sigma\}$. The induced subcomplex or restriction of $\Delta$ on $A \subseteq E$ is defined as $\Delta_{A}=\{\sigma \in \Delta: \sigma \subseteq$ $A\}$. We will write $\Delta \backslash v$ for the restriction of $\Delta$ on the set $E \backslash\{v\}$. A pure simplicial complex $\Delta$ is shellable if there exists a total ordering $\sigma_{1}, \sigma_{2}, \ldots, \sigma_{m}$ of the set of facets of $\Delta$ such that for any given indices $1 \leq i<k \leq m$ there exists $1 \leq j<k$ and $v \in \sigma_{k}$ with $\sigma_{i} \cap \sigma_{k} \subseteq \sigma_{j} \cap \sigma_{k}=\sigma_{k} \backslash\{v\}$. The following lemma is elementary and well known; see, for instance, [15, Section 2].

Lemma 2.1. $\quad$ (i) If $\Delta \backslash v$ is pure of dimension $d$ and shellable and $\mathrm{lk}_{\Delta}(v)$ is pure of dimension $d-1$ and shellable for some $v \in E$ then $\Delta$ is pure of dimension $d$ and shellable.

(ii) The simplicial join of pure shellable complexes is pure and shellable.

When we talk about algebraic or topological properties of an abstract simplicial complex $\Delta$ we refer to those of its Stanley-Reisner ring [17, Chapter II] or geometric realization [8, Section 9], respectively. Thus any pure $d$-dimensional, shellable simplicial complex $\Delta$ is Cohen-Macaulay over $\mathbb{Z}$ and all fields and homotopy equivalent to a wedge of spheres of dimension $d$.

2.2. Generalized cluster complexes. Let $\Phi$ be a finite root system spanning $\mathbb{R}^{n}$, endowed with the standard inner product, and let $\Phi^{+}$be a fixed choice of a positive system with corresponding simple system $\Pi$. We assume temporarily that $\Phi$ is irreducible and let $\Pi=\left\{\alpha_{1}, \ldots, \alpha_{n}\right\}$ be ordered so that for some $1 \leq s \leq n$, each of the sets $\Pi_{+}=\left\{\alpha_{1}, \ldots, \alpha_{s}\right\}$ and $\Pi_{-}=\left\{\alpha_{s+1}, \ldots, \alpha_{n}\right\}$ has pairwise orthogonal elements. Let $\Phi_{\geq-1}=\Phi^{+} \cup(-\Pi)$ and define the involutions $\tau_{ \pm}: \Phi_{\geq-1} \rightarrow \Phi_{\geq-1}$ by

$$
\tau_{\varepsilon}(\alpha)= \begin{cases}\alpha, & \text { if } \alpha \in-\Pi_{-\varepsilon} \\ R_{\varepsilon}(\alpha), & \text { otherwise }\end{cases}
$$

for $\varepsilon \in\{+,-\}$ and $\alpha \in \Phi_{\geq-1}$, where $R_{\varepsilon}$ is the product of the reflections (taken in any order) in the linear hyperplanes in $\mathbb{R}^{n}$ orthogonal to the elements of $\Pi_{\varepsilon}$. The product $\mathcal{R}=\tau_{-} \tau_{+}$, introduced in [13], can be viewed as a deformation of the Coxeter element in the real reflection group corresponding to $\Phi$. Given a nonnegative integer $m$, the set

$$
\Phi_{\geq-1}^{m}=\left\{\alpha^{i}: \alpha \in \Phi^{+} \text {and } 1 \leq i \leq m\right\} \cup(-\Pi)
$$

consists of the negative simple roots and $m$ copies of each positive root, each copy colored with one of $m$ possible colors. Using the convention $\alpha^{1}=\alpha$ for $\alpha \in-\Pi$, the map $\mathcal{R}_{m}: \Phi_{\geq-1}^{m} \rightarrow \Phi_{\geq-1}^{m}$ is defined by

$$
\mathcal{R}_{m}\left(\alpha^{k}\right)= \begin{cases}\alpha^{k+1}, & \text { if } \alpha \in \Phi^{+} \text {and } k<m \\ (\mathcal{R}(\alpha))^{1}, & \text { otherwise. }\end{cases}
$$


It can be shown [11, Theorem 3.4] that there exists a unique symmetric binary relation on $\Phi_{\geq-1}^{m}$, called "compatibility", which has the following two properties:

- $\alpha^{k}$ is compatible with $\beta^{\ell}$ if and only if $\mathcal{R}_{m}\left(\alpha^{k}\right)$ is compatible with $\mathcal{R}_{m}\left(\beta^{\ell}\right)$;

- for $\alpha \in \Pi$, the root $-\alpha$ is compatible with $\beta^{\ell}$ if and only if the simple root expansion of $\beta$ does not involve $\alpha$.

The generalized cluster complex $\Delta^{m}(\Phi)$ is defined in [11, Section 3] is the abstract simplicial complex on the vertex set $\Phi_{\geq-1}^{m}$ which has as its faces the subsets of mutually compatible elements of $\Phi_{\geq-1}^{m}$. If $\Phi$ is a direct product $\Phi_{1} \times \Phi_{2}$ then $\Delta^{m}(\Phi)$ is defined as the simplicial join of $\Delta^{m}\left(\Phi_{1}\right)$ and $\Delta^{m}\left(\Phi_{2}\right)$.

The complex $\Delta^{m}(\Phi)$ is flag by definition and pure of dimension $n-1$ [11, Theorem 3.9]. Following [ 6 , we denote by $\Delta_{+}^{m}(\Phi)$ the induced subcomplex of $\Delta^{m}(\Phi)$ on the set of vertices obtained from $\Phi_{\geq-1}^{m}$ by removing the negative simple roots and call this simplicial complex the positive part of $\Delta^{m}(\Phi)$. For $\alpha \in \Pi$ we denote by $\Phi_{\alpha}$ the standard parabolic root subsystem obtained by intersecting $\Phi$ with the linear span of $\Pi \backslash\{\alpha\}$, endowed with the induced positive system $\Phi_{\alpha}^{+}=\Phi^{+} \cap \Phi_{\alpha}$. Let us summarize the properties of $\Delta^{m}(\Phi)$ which will be of importance for us.

Proposition 2.2. ([1])

(i) If $\Phi$ is a direct product $\Phi_{1} \times \Phi_{2}$ then $\Delta^{m}(\Phi)=\Delta^{m}\left(\Phi_{1}\right) * \Delta^{m}\left(\Phi_{2}\right)$ and $\Delta_{+}^{m}(\Phi)=\Delta_{+}^{m}\left(\Phi_{1}\right) * \Delta_{+}^{m}\left(\Phi_{2}\right)$.

Suppose that $\Phi$ is irreducible.

(ii) If $\alpha \in \Pi$ then $\mathrm{lk}_{\Delta^{m}(\Phi)}(-\alpha)=\Delta^{m}\left(\Phi_{\alpha}\right)$.

(iii) The map $\mathcal{R}_{m}: \Phi_{>-1}^{m} \rightarrow \Phi_{\geq-1}^{m}$ is a bijection with the following properties:

(a) $\sigma \in \Delta^{m}(\Phi)$ if and only if $\mathcal{R}_{m}(\sigma):=\left\{\mathcal{R}_{m}(\alpha): \alpha \in \sigma\right\} \in \Delta^{m}(\Phi)$.

(b) For any $\beta \in \Phi_{\geq-1}^{m}$ there exists $j$ such that $\mathcal{R}_{m}^{j}(\beta) \in(-\Pi)$.

It is not hard to describe $\Delta^{m}(\Phi)$ explicitly when $\Phi$ has type $A_{n-1}$ [11, 19]. Call a diagonal of a convex polygon $\mathbf{P}$ with $m n+2$ vertices $m$-allowable if it divides $\mathbf{P}$ into two polygons, each with number of vertices congruent to $2 \bmod m$. Then vertices of $\Delta^{m}(\Phi)$ biject to the $m$-allowable diagonals of $\mathbf{P}$ so that faces correspond to the sets of pairwise noncrossing diagonals of this kind.

2.3. The reflection length order. We will denote by $R(\alpha)$ the reflection in the linear hyperplane in $\mathbb{R}^{n}$ orthogonal to a nonzero (colored or not) vector $\alpha \in \mathbb{R}^{n}$. Let $W$ be the finite real reflection group generated by the set $T$ of reflections $R(\alpha)$ for $\alpha \in \Phi$. For $w \in W$ we denote by $\ell_{T}(w)$ the smallest integer $r$ such that $w$ can be written as a product of $r$ reflections in $T$. The set $W$ can be partially ordered by letting

$$
u \preceq w \text { if and only if } \ell_{T}(u)+\ell_{T}\left(u^{-1} w\right)=\ell_{T}(w),
$$

in other words if there exists a shortest factorization of $u$ into reflections in $T$ which is a prefix of such a shortest factorization of $v$. The order $\preceq$, called reflection length order or absolute order, turns $W$ into a graded poset having the identity of $W$ as its unique minimal element and rank function $\ell_{T}$. If $\Phi$ has type $A_{n-1}$, so that $W$ can be realized as the symmetric group of permutations of the set $\{1,2, \ldots, n\}$, then one can describe more explicitly these concepts as follows. We have $\ell_{T}(w)=n-c(w)$, where $c(w)$ is the number of cycles of $w$, and $u \preceq w$ if and only if each cycle of $u$ can be obtained from some cycle of $w$ by deleting elements.

We will assume elementary properties of the order $\preceq$; see [9, Section 2] [5], Section 2]. For instance, if $v \preceq u \preceq w$ then $v^{-1} u \preceq v^{-1} w$ and $u v^{-1} \preceq w v^{-1}$. 
2.4. Generalized cluster complexes via the reflection length order. Suppose that $\Phi$ is irreducible and let $N$ denote the cardinality of $\Phi^{+}$. As in Section 2.2. let $\Pi=\left\{\alpha_{1}, \ldots, \alpha_{n}\right\}$ be ordered so that for some $1 \leq s \leq n$ the sets $\Pi_{+}=\left\{\alpha_{1}, \ldots, \alpha_{s}\right\}$ and $\Pi_{-}=\left\{\alpha_{s+1}, \ldots, \alpha_{n}\right\}$ have pairwise orthogonal elements and let

$$
\gamma=R\left(\alpha_{1}\right) R\left(\alpha_{2}\right) \cdots R\left(\alpha_{n}\right)
$$

be a corresponding bipartite Coxeter element of $W$. As in [5, 9] we set $\rho_{i}=$ $R\left(\alpha_{1}\right) R\left(\alpha_{2}\right) \cdots R\left(\alpha_{i-1}\right)\left(\alpha_{i}\right)$ for $i \geq 1$, where the $\alpha_{i}$ are indexed cyclically modulo $n$ (so that $\rho_{1}=\alpha_{1}$ ), and $\rho_{-i}=\rho_{2 N-i}$ for $i \geq 0$ and recall that

$$
\begin{aligned}
& \left\{\rho_{1}, \rho_{2}, \ldots, \rho_{N}\right\}=\Phi^{+}, \\
& \left\{\rho_{N+i}: 1 \leq i \leq s\right\}=\left\{-\rho_{1}, \ldots,-\rho_{s}\right\}=-\Pi_{+}, \\
& \left\{\rho_{-i}: 0 \leq i<n-s\right\}=\left\{-\rho_{N-i}: 0 \leq i<n-s\right\}=-\Pi_{-} .
\end{aligned}
$$

We consider the total order $<$ on the set $\Phi_{\geq-1}=\Phi^{+} \cup(-\Pi)$ defined by

$$
\rho_{-n+s+1}<\cdots<\rho_{0}<\rho_{1}<\cdots<\rho_{N+s} .
$$

This order induces a total order, which we denote again by $<$, on the set of elements of $\Phi_{\geq-1}^{m}$ which are positive roots of some fixed color $i$ simply by forgetting the color. For $\tau=\left\{\tau_{1}, \tau_{2}, \ldots, \tau_{k}\right\} \subseteq \Phi_{\geq-1}^{m}$ such that either $\tau \subseteq(-\Pi)$ or $\tau$ consists of positive roots of the same color, we let

$$
w_{\tau}=R\left(\tau_{k}\right) R\left(\tau_{k-1}\right) \cdots R\left(\tau_{1}\right),
$$

where $\tau_{1}<\tau_{2}<\cdots<\tau_{k}$, with the convention that $w_{\tau}$ is the identity element of $W$ if $\tau=\emptyset$. For any $\sigma \subseteq \Phi_{\geq-1}^{m}$ we let

$$
w_{\sigma}=w_{\sigma^{+}} w_{\sigma^{(m)}} w_{\sigma^{(m-1)}} \cdots w_{\sigma^{(1)}} w_{\sigma^{-}}
$$

where $\sigma^{ \pm}=\left(-\Pi_{ \pm}\right) \cap \sigma$ and for $1 \leq i \leq m, \sigma^{(i)}$ is the set of elements of $\sigma$ which are positive roots of color $i$. The following characterization of $\Delta^{m}(\Phi)$ generalizes that of $\Delta(\Phi)$ given in [9, Section 8].

Theorem 2.3. (20] [21, Section 4.3]) A set $\sigma \subseteq \Phi_{\geq-1}^{m}$ is a face of $\Delta^{m}(\Phi)$ if and only if $w_{\sigma} \preceq \gamma$ and $\ell_{T}\left(w_{\sigma}\right)=|\sigma|$.

Example 2.4. Suppose that $\Phi$ has type $A_{2}$. Then the simple roots $\alpha_{1}, \alpha_{2}$ can be chosen as unit vectors in the Euclidean plane forming an angle of $2 \pi / 3$ and we have $\Pi_{+}=\left\{\alpha_{1}\right\}, \Pi_{-}=\left\{\alpha_{2}\right\}$ and $\Phi^{+}=\left\{\alpha_{1}, \alpha_{2}, \alpha\right\}$, where $\alpha=\alpha_{1}+\alpha_{2}$. Moreover, $W$ is the dihedral group of order 6 generated by the reflections $R\left(\alpha_{1}\right), R\left(\alpha_{2}\right)$ and $R(\alpha)$ and $\gamma=R\left(\alpha_{1}\right) R\left(\alpha_{2}\right)$ is a rotation by $2 \pi / 3$. One can compute easily that $\rho_{1}=\alpha_{1}$, $\rho_{2}=\alpha, \rho_{3}=\alpha_{2}, \rho_{4}=-\alpha_{1}$ and $\rho_{0}=\rho_{6}=-\alpha_{2}$. Therefore the total order (11) on the set $\Phi_{\geq-1}$ is given by

$$
-\alpha_{2}<\alpha_{1}<\alpha<\alpha_{2}<-\alpha_{1} .
$$

Assume that $m=1$ and let $\tau=\left\{\alpha, \alpha_{2}\right\}$. We have $w_{\tau}=R\left(\alpha_{2}\right) R(\alpha)=\gamma$ and hence, according to the condition in Theorem 2.3. $\tau$ must be a facet of $\Delta(\Phi)$. Similarly we find that $\Delta(\Phi)$ must have exactly four more facets, namely $\left\{\alpha_{1}, \alpha\right\},\left\{-\alpha_{2}, \alpha_{1}\right\}$, $\left\{-\alpha_{1},-\alpha_{2}\right\}$ and $\left\{\alpha_{2},-\alpha_{1}\right\}$, corresponding to the factorizations

$$
R(\alpha) R\left(\alpha_{1}\right)=R\left(\alpha_{1}\right) R\left(-\alpha_{2}\right)=R\left(-\alpha_{1}\right) R\left(-\alpha_{2}\right)=R\left(-\alpha_{1}\right) R\left(\alpha_{2}\right)
$$

of $\gamma$. Indeed, $\Delta(\Phi)$ is the one-dimensional simplicial complex having these five twoelement sets as its facets [13. p. 985]. Assume now that $m=2$ and $\tau=\left\{\alpha_{2}^{1}, \alpha_{1}^{2}\right\}$. We have $w_{\tau}=R\left(\alpha_{1}^{2}\right) R\left(\alpha_{2}^{1}\right)=R\left(\alpha_{1}\right) R\left(\alpha_{2}\right)=\gamma$ and hence, by Theorem 2.3, $\tau$ is a 
facet of $\Delta^{2}(\Phi)$. Similarly we find that $\Delta^{2}(\Phi)$ has the following 12 two-element sets as its facets: $\left\{\alpha_{1}^{1}, \alpha^{1}\right\},\left\{\alpha_{1}^{1}, \alpha^{2}\right\},\left\{\alpha_{1}^{2}, \alpha^{2}\right\},\left\{\alpha^{1}, \alpha_{2}^{1}\right\},\left\{\alpha^{1}, \alpha_{2}^{2}\right\},\left\{\alpha^{2}, \alpha_{2}^{2}\right\},\left\{\alpha_{2}^{1}, \alpha_{1}^{2}\right\}$, $\left\{-\alpha_{2}, \alpha_{1}^{1}\right\},\left\{-\alpha_{2}, \alpha_{1}^{2}\right\},\left\{\alpha_{2}^{1},-\alpha_{1}\right\},\left\{\alpha_{2}^{2},-\alpha_{1}\right\}$ and $\left\{-\alpha_{1},-\alpha_{2}\right\}$.

Given $w \preceq \gamma$, we denote by $\Delta_{+}^{m}(w)$ the subcomplex of $\Delta_{+}^{m}(\Phi)$ consisting of those faces $\sigma$ for which $w_{\sigma} \preceq w$. Clearly the dimension of $\Delta_{+}^{m}(w)$ is at most $\ell_{T}(w)-1$. It follows from [5, Lemma 2.1 (iv)] that $\Delta_{+}^{m}(w)$ coincides with the induced subcomplex of $\Delta^{m}(\Phi)$ on the vertex set of positive colored roots $\alpha \in \Phi_{\geq-1}^{m}$ satisfying $R(\alpha) \preceq w$. We will denote this vertex set by $\Phi_{+}^{m}(w)$, so that $\Phi_{+}^{m}(\gamma)$ is the vertex set of $\Delta_{+}^{m}(\Phi)$. When $m=1$ the complex $\Delta_{+}^{m}(w)$, written simply as $\Delta_{+}(w)$, is homeomorphic to a triangulation of a simplex of dimension $\ell_{T}(w)-1$ [9, Corollary 7.7] (in particular it is pure of that dimension) and was shown to be shellable in [5, Theorem 7.1].

Theorem 2.5. ([5, 9]) For any $w \preceq \gamma$ the complex $\Delta_{+}(w)$ is pure of dimension $\ell_{T}(w)-1$ and shellable. Moreover, it is homeomorphic to a ball.

Our definitions of $\Delta_{+}^{m}(w)$ and $\Phi_{+}^{m}(w)$ extend naturally when $\Phi$ is reducible. More precisely, if $\Phi=\Phi_{1} \times \Phi_{2}$ then $\gamma=\gamma_{1} \gamma_{2}$, where $\gamma_{i}$ is a bipartite Coxeter element for the reflection group $W_{i}$ corresponding to $\Phi_{i}$, and $w \preceq \gamma$ if and only if $w=w_{1} w_{2}$ with $w_{1} \preceq \gamma_{1}$ and $w_{2} \preceq \gamma_{2}$. For $\sigma \in \Delta_{+}^{m}(\Phi)$ we can write $\sigma=\sigma_{1} \cup \sigma_{2}$ with $\sigma_{i} \in \Delta_{+}^{m}\left(\Phi_{i}\right)$ for $i=1,2$ and define $w_{\sigma}=w_{\sigma_{1}} w_{\sigma_{2}}$. The subcomplex $\Delta_{+}^{m}(w)$ of $\Delta_{+}^{m}(\Phi)$ consisting of those faces $\sigma$ with $w_{\sigma} \preceq w$ is equal to the simplicial join of $\Delta_{+}^{m}\left(w_{1}\right)$ and $\Delta_{+}^{m}\left(w_{2}\right)$ and has vertex set $\Phi_{+}^{m}(w)=\Phi_{+}^{m}\left(w_{1}\right) \cup \Phi_{+}^{m}\left(w_{2}\right)$.

\section{Proof of Theorem 1.1}

Theorem 1.1 will be derived from the following proposition.

Proposition 3.1. Fix an index $1 \leq j \leq m$.

(i) Given $w \preceq \gamma$, the induced subcomplex of $\Delta_{+}^{m}(w)$ on any subset of its vertex set $\Phi_{+}^{m}(w)$ containing all positive roots in $\Phi_{+}^{m}(w)$ of color $j$ is pure of dimension $\ell_{T}(w)-1$ and shellable.

(ii) The induced subcomplex of $\Delta^{m}(\Phi)$ on any subset of the vertex set of $\Delta^{m}(\Phi)$ containing all positive roots of color $j$ is pure of dimension $n-1$ and shellable.

Proof. (i) In view of the discussion following Theorem 2.5 and Lemma 2.1 (ii), we may assume that $\Phi$ is irreducible. Let $r=\ell_{T}(w)$ and recall that the dimension of $\Delta_{+}^{m}(w)$ does not exceed $r-1$. Let $\Delta_{A}^{m}(w)$ denote the induced subcomplex of $\Delta_{+}^{m}(w)$ on the vertex set $A \subseteq \Phi_{+}^{m}(w)$. Suppose that $A$ contains all positive roots in $\Phi_{+}^{m}(w)$ of color $j$. To simplify notation we set $\Delta=\Delta_{A}^{m}(w)$ and let $\tau$ be any face of $\Delta$. By Theorem 2.3 we have

$$
w_{\tau}=w_{\tau^{(m)}} w_{\tau^{(m-1)}} \cdots w_{\tau^{(1)}} \preceq w
$$

in the notation of (3), and $\ell_{T}\left(w_{\tau}\right)=|\tau|$. It follows that $w_{\tau^{(j)}} \preceq u$ where

$$
u=\left(w_{\tau^{(m)}} \cdots w_{\tau^{(j+1)}}\right)^{-1} w\left(w_{\tau^{(j-1)}} \cdots w_{\tau^{(1)}}\right)^{-1} \preceq w
$$

and $\ell_{T}(u)=r-|\tau|+\left|\tau^{(j)}\right|$. Observe that the set obtained from $\tau^{(j)}$ by uncoloring its elements is a face of $\Delta_{+}(u)$, which is pure of dimension $\ell_{T}(u)-1$ by Theorem 2.5. Hence there exists a set $\sigma^{(j)}$ containing $\tau^{(j)}$ and consisting of $\ell_{T}(u)$ positive roots of color $j$ such that $w_{\sigma^{(j)}}=u$. Setting $\sigma^{(i)}=\tau^{(i)}$ for all $1 \leq i \leq m$ with $i \neq j$, we obtain a subset $\sigma$ of the vertex set of $\Delta_{+}^{m}(\Phi)$ such that $\tau \subseteq \sigma, w_{\sigma}=w$ 
and $|\sigma|=\ell_{T}(w)=r$. It follows from Theorem 2.3 that $\sigma$ is a face of $\Delta^{m}(\Phi)$, and hence of $\Delta_{+}^{m}(w)$. Notice that (i) $A$ contains all positive roots in $\Phi_{+}^{m}(w)$ of color $j$, (ii) $\sigma \backslash \tau=\sigma^{(j)} \backslash \tau^{(j)}$ consists only of such roots and (iii) $\tau \subseteq A$. As a consequence we have $\sigma \subseteq A$. Therefore $\sigma$ is an $(r-1)$-dimensional face of $\Delta$ containing $\tau$. This proves that $\Delta$ is pure of dimension $r-1$.

To prove that $\Delta$ is shellable we proceed by induction on $r$ and the cardinality of $A$, the statement being trivial for $r \leq 1$. Let $S$ be the set of all pairs $(\alpha, i)$ such that $\alpha \in A$ has color $i$, considered with the total ordering in which $(\alpha, i)$ precedes $\left(\alpha^{\prime}, i^{\prime}\right)$ if either $i<i^{\prime}$ or $i=i^{\prime}$ and $\alpha<\alpha^{\prime}$ in the order of roots (11). Theorem 2.3 implies that if $(\alpha, i)$ is the smallest or the largest element of $S$ then $\operatorname{lk}_{\Delta}(\alpha)$ coincides with the complex $\Delta_{B}^{m}(u)$, where $u=w R(\alpha)$ in the former case and $u=R(\alpha) w$ in the latter, and $B=A \cap \Phi_{+}^{m}(u)$. Observe that $B$ contains all roots in $\Phi_{+}^{m}(u)$ of color $j$ and hence, by the previous paragraph and the induction hypothesis, $\operatorname{lk}_{\Delta}(\alpha)$ is pure of dimension $\ell(u)-1=r-2$ and shellable. We now distinguish two cases. If $A$ does not contain any vertices of color other than $j$ then $\Delta$ is combinatorially isomorphic to $\Delta_{+}(w)$ and hence shellable by Theorem 2.5. Otherwise the smallest or the largest pair $(\alpha, i)$ in $S$ satisfies $i \neq j$. We already know that $\operatorname{lk}_{\Delta}(\alpha)$ is pure $(r-2)$-dimensional and shellable. By the same argument $\Delta \backslash \alpha=\Delta_{A \backslash\{\alpha\}}^{m}(w)$ is pure $(r-1)$-dimensional and shellable, since $A \backslash\{\alpha\}$ contains all roots in $\Phi_{+}^{m}(w)$ of color $j$. Part (i) of Lemma 2.1 implies that $\Delta$ is shellable as well.

(ii) Let $\Delta_{A}^{m}(\Phi)$ denote the induced subcomplex of $\Delta^{m}(\Phi)$ on the vertex set $A \subseteq$ $\Phi_{\geq-1}^{m}$. Suppose that $A$ contains all positive roots of color $j$ and set $\Delta=\Delta_{A}^{m}(\Phi)$. We proceed by induction on the rank $n$ of $\Phi$ and the cardinality of $A$ and assume again, as we may, that $\Phi$ is irreducible. If $A$ does not contain any negative simple root then the result follows from the special case $w=\gamma$ of part (i). If $\alpha=-\alpha_{i} \in A$ for some $\alpha_{i} \in \Pi$ then $\Delta \backslash \alpha=\Delta_{A \backslash\{\alpha\}}^{m}(\Phi)$ is pure of dimension $n-1$ and shellable by induction. Moreover, by Proposition 2.2 (ii) we have $\mathrm{lk}_{\Delta}(\alpha)=\Delta_{B}^{m}\left(\Phi_{\alpha}\right)$, where $B=\left(\Phi_{\alpha}\right)_{\geq-1} \cap A$, so that $\operatorname{lk}_{\Delta}(\alpha)$ is pure of dimension $n-2$ and shellable, again by induction. Part (i) of Lemma 2.1 implies that $\Delta$ is pure of dimension $n-1$ and shellable.

Proof of Theorem 1.1. (i) In view of Proposition 2.2(i) and Lemma 2.1(ii) we may assume, once again, that $\Phi$ is irreducible. Suppose that $B \subseteq \Phi_{\geq-1}^{m}$ has at most $m$ elements and let $\Delta_{A}$ denote the induced subcomplex of $\Delta^{m}(\bar{\Phi})$ on the vertex set $A=\Phi_{\geq-1}^{m} \backslash B$. If for some $1 \leq i \leq m$ the set $B$ does not contain any positive root of color $i$ then $\Delta_{A}$ is pure $(n-1)$-dimensional and shellable by Proposition 3.1 (ii). Otherwise let $\beta \in B$ and, by part (b) of Proposition 2.2 (iii), choose $j$ so that $\mathcal{R}_{m}^{j}(\beta) \in(-\Pi)$. Let $B^{\prime}=\mathcal{R}_{m}^{j}(B)$ and $A^{\prime}=\Phi_{\geq-1}^{m} \backslash B^{\prime}$. Part (a) of Proposition 2.2 (iii) implies that $\Delta_{A}$ is combinatorially isomorphic to the induced subcomplex $\Delta_{A^{\prime}}$ of $\Delta^{m}(\Phi)$ on the vertex set $A^{\prime}$. Since $A^{\prime}$ must contain all positive roots of color $i$ for some $1 \leq i \leq m$, the result follows as in the first part of the argument.

(ii) Removing at most $m-1$ vertices from $\Delta_{+}^{m}(\Phi)$ leaves all positive roots of color $i$ in place for at least one index $1 \leq i \leq m$. Hence the result follows from the special case $w=\gamma$ of Proposition 3.1 (i).

Remark 3.2. It is known [7, Theorem 2.1 (a)] that if a simplicial complex $\Delta$ is $k$-Cohen-Macaulay then for any non-maximal face $\sigma \in \Delta$ the complex $\operatorname{lk}_{\Delta}(\sigma)$ has at least $k$ vertices. On the other hand any codimension one face of $\Delta^{m}(\Phi)$ is contained 
in exactly $m+1$ facets [11, Proposition 3.10]. It follows that $m+1$ is the largest integer $k$ for which $\Delta^{m}(\Phi)$ is $k$-Cohen-Macaulay. A similar statement follows for $\Delta_{+}^{m}(\Phi)$ since there exist codimension one faces of $\Delta_{+}^{m}(\Phi)$ which are contained in exactly $m$ facets of $\Delta_{+}^{m}(\Phi)$ (any positive facet of $\Delta^{m}\left(\Phi_{\alpha}\right)$, with $\alpha \in \Pi$, will be such a codimension one face of $\left.\Delta_{+}^{m}(\Phi)\right)$.

The following corollary completes a proof of [6, Conjecture 4.6].

Corollary 3.3. The complex $\Delta_{+}^{m}(\Phi)$ has the homotopy type of a wedge of spheres of dimension $n-1$. If $\Phi$ is irreducible then the number of these spheres is equal to

$$
N^{+}(\Phi, m-1)=\prod_{i=1}^{n} \frac{e_{i}+(m-1) h-1}{e_{i}+1},
$$

where $h$ is the Coxeter number and $e_{1}, e_{2}, \ldots, e_{n}$ are the exponents of $\Phi$.

Proof. The first statement is part of Theorem 1.1 (ii). For the second statement it suffices to show that $\Delta_{+}^{m}(\Phi)$ has reduced Euler characteristic $\tilde{\chi}\left(\Delta_{+}^{m}(\Phi)\right)=$ $(-1)^{n-1} N^{+}(\Phi, m-1)$. This was shown in [6] for irreducible root systems $\Phi$ of classical type with a proof that can be extended to any type. We give the details here for the record. To match the notation of [6] we write $\Phi=\Phi_{I}$, where $I$ is an index set in bijection with $\Pi$, and denote by $\Phi_{J}$ the parabolic root subsystem corresponding to $J \subseteq I$. We also define $N^{+}\left(\Phi_{I}, m\right)$ multiplicatively on the irrecucible components of $\Phi_{I}$, if $\Phi_{I}$ is reducible. As was mentioned in Remark 3 of [6, Section 7], Theorem 3.7 in [11] implies that equation (6) in [6] holds without any restriction on $\Phi$. Multiplying both sides with $(-1)^{k-1}$ and summing over $k$ we get

$$
(-1)^{|I|-1} \tilde{\chi}\left(\Delta^{m}\left(\Phi_{I}\right)\right)=\sum_{J \subseteq I}(-1)^{|J|-1} \tilde{\chi}\left(\Delta_{+}^{m}\left(\Phi_{J}\right)\right) .
$$

On the other hand

$$
(-1)^{|I|-1} \tilde{\chi}\left(\Delta^{m}\left(\Phi_{I}\right)\right)=\sum_{J \subseteq I} N^{+}\left(\Phi_{J}, m-1\right)
$$

by Propositions 11.1 and 12.3 in [11. Inclusion-exclusion implies the desired formula for $\tilde{\chi}\left(\Delta_{+}^{m}(\Phi)\right)$.

Remark 3.4. Using results of [5, Section 4] (in particular [5, Corollary 4.6]) it is possible to show that removing the vertices of $\Delta_{+}(w)$ in the order prescribed by (11) gives a vertex decomposition of $\Delta_{+}(w)$, in the sense of [15, for any $w \preceq \gamma$. It follows from the proofs of our results in this section that $\Delta^{m}(\Phi)$ and $\Delta_{+}^{m}(\Phi)$ are vertex decomposable as well.

\section{Generalized noncrossing Partitions}

In this section we discuss some connections with the posets of generalized noncrossing partitions, defined and studied by D. Armstrong [2. Let $\gamma \in W$ be any Coxeter element and $\mathbf{L}_{W}^{(m)}$ denote the set of $m$-tuples $\left(w_{1}, w_{2}, \ldots, w_{m}\right)$ of elements of $W$ satisfying $w_{1} w_{2} \cdots w_{m} \preceq \gamma$ and

$$
\ell_{T}\left(w_{1} w_{2} \cdots w_{m}\right)=\ell_{T}\left(w_{1}\right)+\ell_{T}\left(w_{2}\right)+\cdots+\ell_{T}\left(w_{m}\right) .
$$

We partially order this set by letting

$$
\left(u_{1}, u_{2}, \ldots, u_{m}\right) \preceq\left(w_{1}, w_{2}, \ldots, w_{m}\right) \text { if } u_{i} \preceq w_{i} \text { for } 1 \leq i \leq m
$$


(this is the dual to the partial order defined in [2]). The isomorphism type of $\mathbf{L}_{W}^{(m)}$ does not depend on the choice of $\gamma$.

Remark 4.1. We have $\left(u_{1}, u_{2}, \ldots, u_{m}\right) \in \mathbf{L}_{W}^{(m)}$ whenever $\left(w_{1}, w_{2}, \ldots, w_{m}\right) \in \mathbf{L}_{W}^{(m)}$ and $u_{i} \preceq w_{i}$ for all $1 \leq i \leq m$.

The poset $\mathbf{L}_{W}^{(m)}$ has a unique minimal element $\hat{0}$ and is graded with rank function $\ell_{T}\left(w_{1}, w_{2}, \ldots, w_{m}\right):=\ell_{T}\left(w_{1} w_{2} \cdots w_{m}\right)$. Now let $\gamma$ be as in Section 2.4. By Theorem 2.3 we have

$$
f(\sigma)=\left(w_{\sigma^{(m)}}, w_{\sigma^{(m-1)}}, \ldots, w_{\sigma^{(1)}}\right) \in \mathbf{L}_{W}^{(m)}
$$

for any face $\sigma$ of $\Delta_{+}^{m}(\Phi)$. Recall that the upper truncation $P^{\leq k}$ of a graded poset $P$ is the induced subposet on the set of elements of rank at most $k$ (see [18, Chapter 3] for basic background on partially ordered sets). If $\Delta$ is a simplicial complex then a map $g: \Delta-\{\emptyset\} \rightarrow P$ is order preserving if $g(\tau) \leq g(\sigma)$ holds in $P$ whenever $\tau \subseteq \sigma$ are nonempty faces of $\Delta$. In what follows, we say that a poset $P$ has a certain topological property if the geometric realization of the simplicial complex of chains in $P$ [8, Section 9] has the same property.

Theorem 4.2. The map

$$
f: \Delta_{+}^{m}(\Phi)-\{\emptyset\} \rightarrow \mathbf{L}_{W}^{(m)}-\hat{0}
$$

is well defined and order preserving and induces a homotopy equivalence between the skeleton $\left(\Delta_{+}^{m}(\Phi)\right) \leq k-1$ and $\left(\mathbf{L}_{W}^{(m)}\right) \leq k-\hat{0}$ for all $1 \leq k \leq n$.

In particular, $\mathbf{L}_{W}^{(m)}-\hat{0}$ is homotopy equivalent to $\Delta_{+}^{m}(\Phi)$.

Proof. Let us write $\Delta=\Delta_{+}^{m}(\Phi)$ and $\mathbf{L}=\mathbf{L}_{W}^{(m)}$, to simplify notation. That the map $f$ is well defined and order preserving follows from Theorem 2.3. Moreover we have $\ell_{T}(f(\sigma))=|\sigma|$ for all $\sigma \in \Delta$ and hence $f$ induces a well defined order preserving map from $\Delta^{\leq k-1}$ to $\mathbf{L} \leq k-\hat{0}$ for all $1 \leq k \leq n$. To complete the proof, by Quillen's Fiber Theorem [8, Theorem 10.5 (i)] it suffices to show that $f^{-1}\left(\mathbf{L}_{\leq x}\right)$ is contractible for all $x \in \mathbf{L}-\hat{0}$. Indeed, it follows directly from the definitions and Remark 4.1 that if $x=\left(w_{1}, w_{2}, \ldots, w_{m}\right)$ then $f^{-1}\left(\mathbf{L}_{\leq x}\right)$ is combinatorially isomorphic to the simplicial join of the complexes $\Delta_{+}^{m}\left(w_{i}\right)$ for $1 \leq i \leq m$, which is contractible by Theorem 2.5

Remark 4.3. (i) It follows from Corollary 3.3 and Theorem 4.2 that $\mathbf{L}_{W}^{(m)}-\hat{0}$ is homotopy equivalent to a wedge of $(n-1)$-dimensional spheres. With some more work, and using the previous statement as well as the main result of [4, one can show that the poset $\mathbf{L}_{W}^{(m)} \cup \hat{1}$, obtained from $\mathbf{L}_{W}^{(m)}$ by adjoining a maximum element $\hat{1}$, is homotopy Cohen-Macaulay (hence Cohen-Macaulay). We omit the details since a stronger statement, namely that $\mathbf{L}_{W}^{(m)} \cup \hat{1}$ is shellable, has been proved by D. Armstrong and H. Thomas [2, Theorem 3.7.2].

(ii) The proof of Theorem 4.2 shows that any order ideal $\mathcal{I}$ in $\mathbf{L}_{W}^{(m)}-\hat{0}$ is homotopy equivalent to the subcomplex $f^{-1}(\mathcal{I})$ of $\Delta_{+}^{m}(\Phi)$.

(iii) In the case $m=1$ and $k=n-1$, Theorem 4.2 gives a new proof of the fact [4. Corollary 4.4] that the Möbius number $\mu(\hat{0}, \gamma)$ of $\mathbf{L}_{W}^{(1)}$ is equal, up to the sign $(-1)^{n}$, to the number of facets of $\Delta_{+}(\Phi)$ (positive clusters associated to $\Phi$ ). 
Acknowledgements. The authors thank the anonymous referee for several helpful suggestions.

\section{REFERENCES}

[1] D. Armstrong, Braid groups, clusters and free probability: an outline from the AIM Workshop, January 2005, available at http://www. aimath.org/WWN/braidgroups/

[2] D. Armstrong, Generalized noncrossing partitions and combinatorics of Coxeter groups, Ph.D thesis, Cornell University, 2006.

[3] C.A. Athanasiadis, On a refinement of the generalized Catalan numbers for Weyl groups, Trans. Amer. Math. Soc. 357 (2005), 179-196.

[4] C.A. Athanasiadis, T. Brady and C. Watt, Shellability of noncrossing partition lattices, Proc. Amer. Math. Soc. 135 (2007), 939-949, math.C0/0503007

[5] C.A. Athanasiadis, T. Brady, J. McCammond and C. Watt, $h$-Vectors of generalized associahedra and noncrossing partitions, Int. Math. Res. Not. vol. 2006, Article 69705, 28pp, math.CO/0602293

[6] C.A. Athanasiadis and E. Tzanaki, On the enumeration of positive cells in generalized cluster complexes and Catalan hyperplane arrangements, J. Algebraic Combin. 23 (2006), 355-375, math.CO/0605685

[7] K. Baclawski, Cohen-Macaulay connectivity and geometric lattices, European J. Combin. 3 (1982), 293-305.

[8] A. Björner, Topological methods, in Handbook of combinatorics (R.L. Graham, M. Grötschel and L. Lovász, eds.), North Holland, Amsterdam, 1995, pp. 1819-1872.

[9] T. Brady and C. Watt, Lattices in finite real reflection groups, Trans. Amer. Math. Soc. (to appear), math.CO/0501502

[10] S. Fomin and N. Reading, Root systems and generalized associahedra, in Geometric Combinatorics, IAS/Park City Mathematics Series (to appear), math.C0/0505518.

[11] S. Fomin and N. Reading, Generalized cluster complexes and Coxeter combinatorics, Int. Math. Res. Not. 44 (2005), 2709-2757, math.CO/0505085

[12] S. Fomin and A.V. Zelevinsky, Cluster algebras I: Foundations, J. Amer. Math. Soc. 15 (2002), 497-529, math.RT/0104151.

[13] S. Fomin and A.V. Zelevinsky, $Y$-systems and generalized associahedra, Ann. of Math. 158 (2003), 977-1018, hep-th/0111053.

[14] G. Kalai, personal communication, 2006.

[15] J.S. Provan and L.J. Billera, Decompositions of simplicial complexes related to diameters of convex polyhedra, Math. Operations Research 5 (1980), 576-594.

[16] E. Swartz, g-elements, finite buildings and higher Cohen-Macaulay connectivity, J. Combin. Theory Series A 113 (2006), 1305-1320, math.C0/0512086

[17] R.P. Stanley, Combinatorics and Commutative Algebra, Progress in Mathematics 41, Birkhäuser, Boston, first edition, 1983; second edition, 1996.

[18] R.P. Stanley, Enumerative Combinatorics, vol. 1, Wadsworth \& Brooks/Cole, Pacific Grove, CA, 1986; second printing, Cambridge University Press, Cambridge, 1997.

[19] E. Tzanaki, Polygon dissections and some generalizations of cluster complexes, J. Combin. Theory Series A 113 (2006), 1189-1198, math.C0/0501100

[20] E. Tzanaki, Faces of generalized cluster complexes and noncrossing partitions, preprint, 2006, $15 \mathrm{pp}$, math.CO/0605785

[21] E. Tzanaki, Combinatorics of generalized cluster complexes and hyperplane arrangements (in greek), Doctoral Dissertation, University of Crete, 2007.

Department of Mathematics (Division of Algebra-Geometry), University of Athens, Panepistimioupolis, 15784 Athens, Greece

E-mail address: caath@math.uoa.gr

Department of Mathematics, University of Crete, 71409 Heraklion, Crete, Greece

E-mail address: etzanaki@math.uoc.gr 